\title{
Medium and Large Vector-Based SVPWM Technique for Five-Phase Two-Level Inverter
}

\author{
Erkan Deniz ${ }^{1 *}$ \\ ${ }^{1 *}$ Firat University, Technology Faculty, Electrical-Electronics Engineering, 23119, Elazig, Turkey. (e-mail: edeniz@ firat.edu.tr).
}

\section{ARTICLE INFO}

Received: Nov., 27. 2021

Revised: Dec., 07. 2021

Accepted: Dec., 08. 2021

\section{Keywords:}

Discontinuous Space Vector PWM

Five Phase Inverter

Large and Medium Vectors

THD Analysis

Corresponding author: Erkan Deniz

ISSN: 2536-5010 | e-ISSN: 2536-5134

\section{ABSTRACT}

In recent years, interest in the use of multi-phase (more than three-phase) dc/ac inverters in applications such as electric vehicles, electric traction, more electric aircraft, ship propulsion, aerospace applications, etc. has been increasing day by day. The paper presents the Discontinuous Space Vector Pulse Width Modulation (DSVPWM) technique for control of a five-phase two-level inverter. The five-phase DSVPWM algorithm uses zero, medium and large vectors combination. When calculating vectors dwell times, calculations are made by directing the $\mathrm{V}_{\text {ref }}$ to Sector-1 regardless of which sector it falls into. Thus, complex calculations are reduced. DSVPWM controlled five-phase two-level inverter that feeds an RL load is simulated using MATLAB/Simulink-Simscape blocks. To demonstrate the effectiveness of the used DSVPWM algorithm, five-phase inverter's output voltages, current waveforms and their FFT analysis are given for different modulation indexes and switching frequencies.

DOI: https://doi.org/10.36222/ejt.1029249

\section{INTRODUCTION}

Conventional variable speed electric drives, widely used in industry, consist of a three-phase power electronic converter and ac motor/motors. Since the power electronic converter is an intermediate device that separates the source and the machine, the number of phases of the machine in variable speed electric drives does not necessarily need to be limited to three [1]. The increase in the switching frequencies of power electronic switches and the decrease in their prices have made both the control of electric motors easy and flexible, and the number of phases of the machine has also become a design parameter [2]. Today, multi-phase motors larger than threephase have become a promising technology, especially for medium and high power applications. There is a high possibility of the coincidence of the poles in motors with even phase numbers, which reduces the motor's performance. Therefore, motors with odd phase numbers are preferred in practice [3]. The lowest multi-phase motors most commonly used in this case are the five-phase ones. As the number of phases increases in electric motors, higher efficiency and torque density are obtained while the amplitude of the torque fluctuations decreases and its frequency increases [4]. In addition, the stator currents per phase and thus the stator copper losses decrease, while the fault tolerance of the motor also increases. An $m$-phase motor can operate with the $(m-3)$ phase in the event of a fault, provided that the non-faulted phases are designed to withstand transient currents. For example, in the event of a fault, a five-phase motor can continue to operate by creating a rotating magnetic field with its solid two phases. This high fault tolerance has increased the interest in using multiphase motors in sensitive applications that are very popular today, such as electric vehicles, electric locomotives, ships and space applications $[5,6]$. On the other hand, three-phase machines have two stator winding types of triangle and star, while different winding configurations occur in multi-phase machines. For example, for a 5-phase motor, there are star, pentagonal and five-pointed star winding types. As a result, as the number of phases increases, the increase in the stator winding connection types allows obtaining motors with different speed-torque characteristics [6,7].

Utilizing the potential of an m-phase motor is only possible if a power electronic converter with an $\mathrm{m}$-phase feeds the motor. Because the conventional electrical grid does not have more than three phases [8]. As the number of phases increases, the power on one leg of the inverter decreases. In this case, large powered inverters for a multi-phase drive system can be realized with lower-rated semiconductor devices. It has also been observed that the overall performance and especially fault tolerance of multi-phase systems are better compared to conventional three-phase system drives [9]. In multi-phase inverters, the number of switching increases with the increasing number of switches. For example, 32 switching states and the associated voltage space vectors form in a five-phase inverter with ten switches. In this way, five-phase inverters allow better adjustment of flux and torque in the motor compared to threephase inverters. In multi-phase drive systems, two types of pulse width modulation (PWM) strategies are commonly used 
to obtain a voltage of variable amplitude and variable frequency from a voltage source inverter. These are carrierbased sinusoidal PWM (SPWM) and space vector PWM (SVPWM). Compared to SPWM, the SVPWM technique has advantages such as easy implementation for digital applications, more efficient use of dc-link voltage and lower THD in output waveforms [10].

There are two switching sequences for SVPWM. One of them is continuous and the other is discontinuous SVPWM (DSVPWM) [11]. In continuous SVPWM, half of a switching period starts with a zero vector (00000) and ends with another zero vector (11111) to obtain a symmetrical switching signal. There are also active vectors in the switching sequence. In DSVPWM, it is aimed to reduce switching losses and total harmonic distortion (THD) in output current by making less number of switching. This can be done by arranging the placement of the zero voltage vectors in a switching period. Reducing switching losses in multi-phase drive systems means increasing energy efficiency, as the application areas are high power range and the number of switches is more [12]. Six different DSVPWM techniques have been used in the literature. The method where $\mathrm{T}_{31}(11111)$ is kept zero for a switching period is called DPWMMIN and the method where $\mathrm{T}_{0}(00000)$ is kept zero is called DPWMMAX. Other methods called DPWM0, DPWM1, DPWM2 and DPWM3 organize zero space vectors in various ways in sectors. Especially at low modulation indices, the DPWMAX scheme offers the lowest THD value $[12,13]$.

In this study, the DSVPWM technique is used to control a five-phase two-level inverter. In this way, the switching status of one leg of the inverter is kept constant throughout the switching period. The DPWMMAX scheme of DSVPWM is proposed to obtain low THD output from the inverter output even at low modulation indices. This algorithm was developed as software using S-Function blocks in MATLAB/Simulink environment. In order to reduce the computational complexity, the reference vector is always directed to sector-1, regardless of sector number. The control of the five-phase inverter created with MATLAB/Simscape blocks was made using the developed DSVPWM algorithm. Inverter output phase and phase-to-phase voltages and phase currents are given for different modulation indexes and switching frequencies. The simulation results obtained show the accuracy of the algorithm. This article is organized as follows: The advantages of a fivephase source over a three-phase are given in section II. In Chapter III, the five-phase SVPWM algorithm is given in detail. A discontinuous DPWMMAX algorithm is proposed for the control of the inverter. The simulation results are given in Section-4.

\section{COMPARISON OF THREE PHASE AND FIVE PHASE SOURCES}

For a balanced Y-connected three-phase system shown in Figure 1(a), the phase voltages have the same amplitude $\left(\mathrm{V}_{\mathrm{a}}=\mathrm{V}_{\mathrm{b}}=\mathrm{V}_{\mathrm{c}}=\mathrm{V}_{\mathrm{L}}\right)$ and have a phase difference of $120^{\circ}$ between them. The phase-to-phase voltages are $\mathrm{V}_{\mathrm{ab}}=\mathrm{V}_{\mathrm{bc}}=\mathrm{V}_{\mathrm{ca}}=\mathrm{V}_{\mathrm{LL}}=$ $\sqrt{3} \mathrm{~V}_{\mathrm{L}}$ and the phase current is equal to the phase-to-phase current $\left(\mathrm{I}_{\mathrm{LL}}=\mathrm{I}_{\mathrm{L}}\right)$. In this case, the three-phase power can be calculated using of Eqs. (1).

$$
P_{3}=3 V_{L} I_{L} \cos \varphi=\sqrt{3} V_{L L} I_{L L} \cos \varphi
$$

For a balanced Y-connected five-phase system shown in Figure 1(b), the phase voltages have the same amplitude $\left(\mathrm{V}_{\mathrm{a}}=\mathrm{V}_{\mathrm{b}}=\mathrm{V}_{\mathrm{c}}=\mathrm{V}_{\mathrm{d}}=\mathrm{V}_{\mathrm{e}}=\mathrm{V}_{\mathrm{L}}\right)$ and have a phase difference of $72^{\circ}$ between them. For a five-phase system, there are five phase-tophase voltages $\left(\mathrm{V}_{\mathrm{ab}}=\mathrm{V}_{\mathrm{bc}}=\mathrm{V}_{\mathrm{cd}}=\mathrm{V}_{\mathrm{de}}=\mathrm{V}_{\mathrm{ea}}=\mathrm{V}_{\mathrm{LL} 1}\right)$ with equal amplitude formed by adjacent phases, and also five phase-tophase voltages $\left(\mathrm{V}_{\mathrm{ac}}=\mathrm{V}_{\mathrm{bd}}=\mathrm{V}_{\mathrm{ce}}=\mathrm{V}_{\mathrm{da}}=\mathrm{V}_{\mathrm{eb}}=\mathrm{V}_{\mathrm{LL} 2}\right)$ with equal amplitudes formed by non-adjacent phases. In addition, the phase current is equal to the phase-to-phase current $\left(\mathrm{I}_{\mathrm{LL}}=\mathrm{I}_{\mathrm{L}}\right)$. The amplitudes of these phase-to-phase voltages can be calculated by Eqs. (2)-(3), respectively. As can be seen from the equations, the amplitudes of the phase-to-phase voltages formed by the adjacent phases are smaller than the three-phase system, while the amplitudes of the phase-to-phase voltages formed by the non-adjacent phases are larger. In this case, the five-phase power can be calculated by Eqs. (4) $[14,15]$.

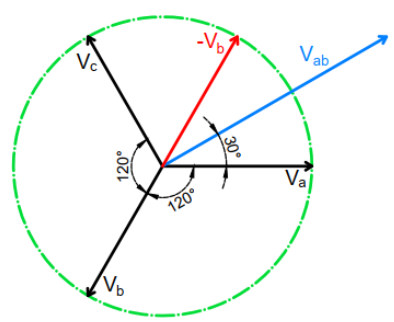

(a)

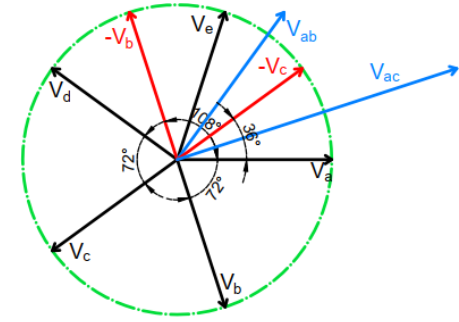

(b)
Figure 1. Phasor representation of voltages; (a) Three-phase, (b) Five-phase

$$
\begin{aligned}
& \left|V_{L L 1}\right|=\left|V_{a b}\right|=\sqrt{\left|V_{a}\right|^{2}+\left|V_{b}\right|^{2}+\left|V_{a}\right|\left|V_{b}\right| \cos (3 \pi / 5)}=1.1756 * V_{L} \\
& \left|V_{L L 2}\right|=\left|V_{a c}\right|=\sqrt{\left|V_{a}\right|^{2}+\left|V_{c}\right|^{2}+\left|V_{a}\right|\left|V_{c}\right| \cos (\pi / 5)}=1.9025^{*} V_{L} \\
& P_{5}=5 V_{L} I_{L} \cos \varphi=(5 / 1.1756) V_{L L} I_{L L} \cos \varphi=4.25 V_{L L} I_{L L} \cos \varphi
\end{aligned}
$$

Considering Eqs. (1) and Eqs. (4) together, the currents drawn from a five-phase source for the same phase voltages and same output power will be approximately $40 \%$ less than a three-phase source. In this case, the reduction of current per phase for a five-phase inverter allows the use of smaller rated semiconductor switches, which reduces cost.

\section{SVPWM TECHNIQUE FOR FIVE-PHASE VOLTAGE SOURCE INVERTER}

The circuit of a five-phase two-level voltage source inverter is shown in Figure 2. This circuit consists of ten IGBTs and freewheeling diodes. There are $2^{5}=32$ possible switching states depending on whether the upper five switches on the circuit are turned on or turned off. These states determine the output voltage. In addition, the inverter generates 32 different voltage vectors for these 32 switching states. The 32 different switching states in a five-phase inverter circuit and their corresponding voltage vectors are given in Table 1. A switching state of " 1 " indicates that the upper switch is turned on, and " 0 " means that the lower switch of the same phase leg is turned off. Two of these vectors are called zero state vectors $\left(\mathrm{V}_{0}\right.$ and $\left.\mathrm{V}_{31}\right)$ and the remaining thirty are called active state vectors $\left(\mathrm{V}_{1}-\mathrm{V}_{30}\right)$. When each switching state in Table 1 is substituted in Eqs. (5), the vector corresponding to the switching state is defined in space. Here, $y$ is the number of the vector and $\underline{a}=e^{j \alpha}, \alpha=2 \pi / 5$ [16]. 


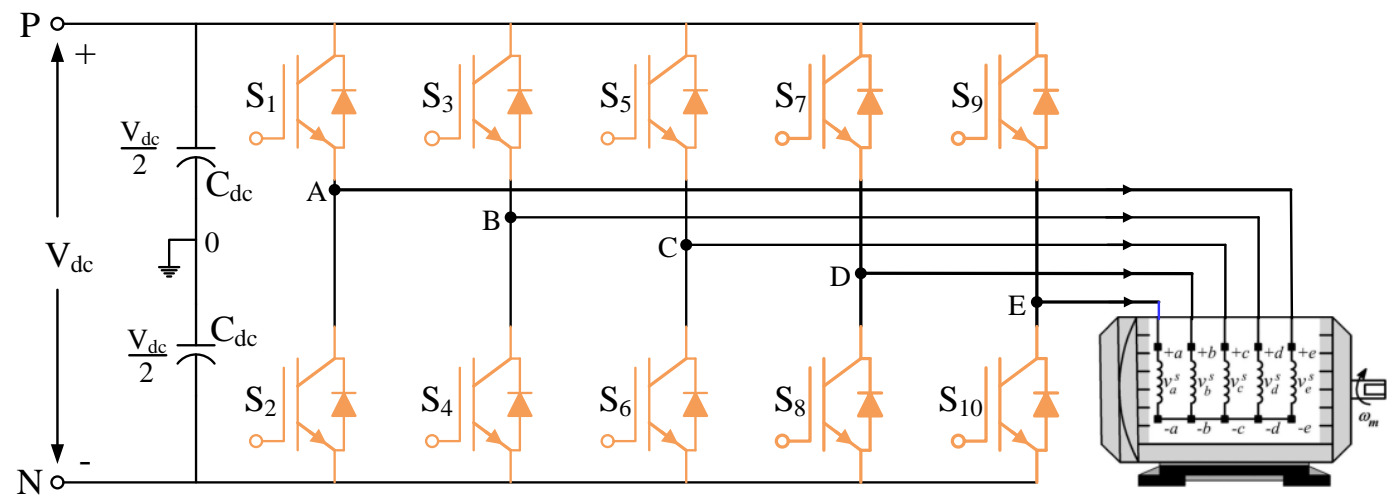

Figure 2. Five-phase two-level voltage source inverter circuit

TABLE I

Active Voltage Vectors and CoRResponding Switching States

\begin{tabular}{|c|c|c|}
\hline $\begin{array}{c}\text { Large Vectors } \\
0.6472^{*} \mathrm{~V}_{\mathrm{dc}} \\
\end{array}$ & $\begin{array}{c}\text { Medium Vectors } \\
0.4^{*} V_{\mathrm{dc}} \\
\end{array}$ & $\begin{array}{c}\text { Small Vectors } \\
0.2472^{*} V_{\mathrm{dc}} \\
\end{array}$ \\
\hline$\vec{V}_{1}[11001] \quad \vec{V}_{6}[00110]$ & $\vec{V}_{11}[10000] \vec{V}_{16}[01111]$ & $\vec{V}_{21}[01001] \quad \vec{V}_{26}[10110]$ \\
\hline$\vec{V}_{2}[11000] \quad \vec{V}_{7}[0$ & $\vec{V}_{12}[11101] \vec{V}_{17}[00010]$ & $\vec{V}_{22}[11010] \vec{V}_{27}[00101]$ \\
\hline$\vec{V}_{3}[11100] \quad \vec{V}_{8}[00011]$ & $\vec{V}_{13}[01000] \vec{V}_{18}[10111]$ & $\vec{V}_{23}[10100] \quad \vec{V}_{28}[01011]$ \\
\hline$\vec{V}_{4}[01100] \quad \vec{V}_{9}[10011]$ & $\vec{V}_{14}[11110] \vec{V}_{19}[00001]$ & $\vec{V}_{24}[01101] \vec{V}_{29}[10010]$ \\
\hline$\vec{V}_{5}[01110] \quad \vec{V}_{10}[10001]$ & $\vec{V}_{15}[00100] \vec{V}_{20}[11011]$ & ]$\vec{V}_{25}[01010] \vec{V}_{30}[10101]$ \\
\hline
\end{tabular}

$$
\vec{V}_{y}=\underline{V}_{\alpha \beta}^{i n v}=\frac{2}{5}\left(V_{a}+\underline{a} V_{b}+\underline{a}^{2} V_{c}+\underline{a}^{3} V_{d}+\underline{a}^{4} V_{e}\right)
$$

Considering a five-phase system, the inverter voltage vectors must be represented in a five-dimensional space. Such a space can be divided into two two-dimensional subspaces $(\alpha-\beta$ and $x$ $y$ ) and one one-dimensional subspace (zero sequences). For a star-connected system with an isolated neutral point, the zero sequence is not used. Therefore, it is sufficient to consider only two-dimensional subspaces $(\alpha-\beta$ and $x-y)$. The $\mathrm{x}-\mathrm{y}$ subspace is generally not used because it is a source of third-order harmonics. When 32 voltage vectors are placed on the $\alpha-\beta$ plane, a vector space containing three concentric decagons is formed as shown in Figure 3. There is three decagons with ten sectors of $36^{\circ}$ each. Vectors in the innermost decagon are called small vectors, vectors in the middle decagon are called medium vectors, and vectors in the outermost decagon are called large vectors. The space vectors of the outermost and innermost decagon are formed when the upper three switches are on and the two switches are off in the inverter circuit. Thus, the innermost space vectors in the $\alpha-\beta$ plane are redundant and are usually ignored. Space vectors in the middle decagon are formed when the upper four switches are on and one switch is off in the inverter circuit [17]. The five-phase voltages desired to be obtained from the output of the inverter can be expressed with a reference voltage vector $\mathrm{V}_{\text {ref }}$ in the $\alpha-\beta$ subspace as seen in Figure 3. The amplitude and angle of the reference vector are obtained using Eqs. (6), (7) and (8). Where, $\underline{a}=e^{j \alpha}, \alpha=$ $2 \pi / 5$.

$$
\left[\begin{array}{c}
V_{\alpha} \\
V_{\beta}
\end{array}\right]=\left[\begin{array}{ccccc}
1 & \cos \alpha & \cos 2 \alpha & \cos 3 \alpha & \cos 4 \alpha \\
0 & -\sin \alpha & -\sin 2 \alpha & -\sin 3 \alpha & -\sin 4 \alpha
\end{array}\right]\left[\begin{array}{c}
V_{a} \\
\vdots \\
V_{e}
\end{array}\right]
$$

$$
\begin{gathered}
V_{r e f}=\sqrt{\left(V_{\alpha}\right)^{2}+\left(V_{\beta}\right)^{2}} \\
\theta=\tan ^{-1}\left(V_{\beta} / V_{\alpha}\right)
\end{gathered}
$$

The phase voltages in Eqs. (6) are defined as follows ;

$$
\begin{array}{ll}
V_{a}=V_{m} \sin (\omega t) & V_{b}=V_{m} \sin (\omega t-2 \pi / 5) \\
V_{c}=V_{m} \sin (\omega t-4 \pi / 5) & V_{d}=V_{m} \sin (\omega t-6 \pi / 5) \\
V_{e}=V_{m} \sin (\omega t-8 \pi / 5) &
\end{array}
$$

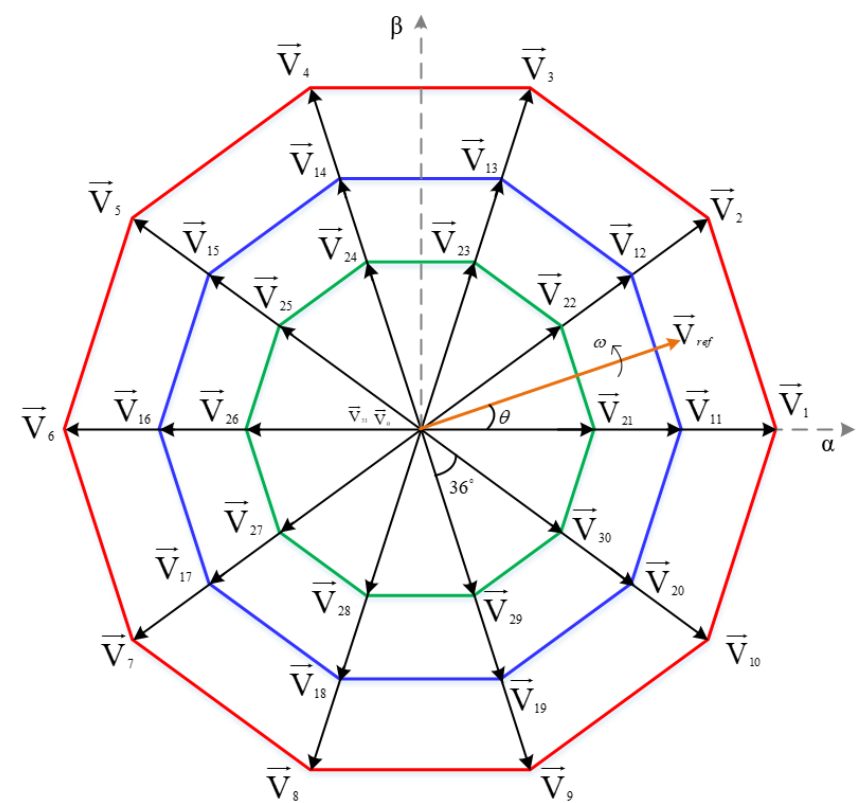

Figure 3. Vector space of five-phase two-level inverter in the $\alpha-\beta$ plane

The sequence followed when generating SVPWM switching signals for a five-phase inverter is similar to that of a three-phase. The angle used in determining the sector numbers is $36^{\circ}$ and its multiples. Two different switching schemes are commonly used when calculating the dwell times of vectors according to the sector in which the reference vector is located. The first is to establish the volt-second balancing equation using only zero vectors and large vectors in the sector. The second is to establish the volt-second balancing equation by using two medium and two large vectors in the sector in addition to the zero vector $[9,18]$. The first scheme causes undesired low-order harmonics in the output phase voltage of the inverter. The number of space vectors used in a switching period should be kept equal to the inverter phase number to eliminate this disadvantage. This means that four active vectors 
must be used in each switching period [19]. The use of two adjacent middle vectors together with two large vectors in each switching period makes it possible to maintain zero mean values in the second subspace $(x-y)$ and obtain a sinusoidal output. The use of four active space vectors in a switching period requires the calculation of the four dwell-times of the large and medium vectors, represented by $T_{a l}, T_{b l}, T_{a m}, T_{b m}$ in Figure 4. If $\mathrm{V}_{\text {ref }}$ is in Sector-1, the voltage-time balancing equation is as follows [16].

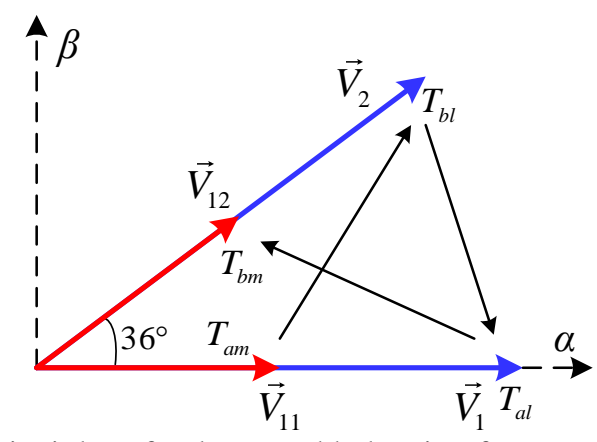

Figure 4. Principles of volt-second balancing for sector-1

$$
\begin{aligned}
& \vec{V}_{r e f} * T_{s}=\vec{V}_{a l} * T_{a l}+\vec{V}_{a m} * T_{a m}+\vec{V}_{b l} * T_{b l}+\vec{V}_{b m} * T_{b m}+\vec{V}_{0} * T_{0} \\
& T_{s}=T_{a l}+T_{a m}+T_{b l}+T_{b m}+T_{0}
\end{aligned}
$$

Here $\mathrm{T}_{0}$ represents the switching times of the zero vectors. The lengths of the large, medium and zero vectors in Figure 4 are given in Eqs. (11).

$$
\begin{aligned}
& \left|\underline{V}_{a m}\right|=\left|\underline{V}_{b m}\right|=\left|\underline{V}_{m}\right|=(2 / 5) * V_{d c} \\
& \left|\underline{V}_{a l}\right|=\left|\underline{V}_{b l}\right|=\left|\underline{V}_{l}\right|=(2 / 5) * V_{d c} * 2 \cos (\pi / 5) \\
& \left|\underline{V}_{0}\right|=\left|\underline{V}_{31}\right|=0
\end{aligned}
$$

In this SVPWM scheme, the ratio of the dwell times of the large and medium vectors is kept equal to the ratio of their lengths and expressed as follows.

$$
\frac{T_{l}}{T_{m}}=\frac{\left|\underline{V}_{l}\right|}{\left|\underline{V}_{m}\right|}=\tau=2 \cos (\pi / 5)=1.618
$$

After substituting the expressions in Eqs. (11) and Eqs. (12) in Equation 10, if the expressions are divided into real and imaginary parts, the general expression of the dwell times for all sectors is obtained as in Eqs. (13) and Eqs. (14) [17]. Here $\mathrm{k}=1,2, \ldots 10$ denotes the sector value. $\left|\underline{V}_{r e f}\right|$ symbolizes the amplitude of the reference space vector and $\theta$ its angle. The indices ' $l$ ' and ' $m$ ' are used to represent large and medium vectors, respectively.

$$
\begin{aligned}
T_{a m} & =\frac{\left|\underline{V}_{r e f}\right| \sin \left(\frac{k \pi}{5}-\theta\right)}{\left|\underline{V}_{m}\right| \sin \left(\frac{\pi}{5}\right)}\left[\frac{1}{1+\tau^{2}}\right] T_{s} \\
T_{b m} & =\frac{\left|\underline{V}_{r e f}\right| \sin \left(\theta-\frac{(k-1) \pi}{5}\right)}{\left|\underline{V}_{m}\right| \sin \left(\frac{\pi}{5}\right)}\left[\frac{1}{1+\tau^{2}}\right] T_{s}
\end{aligned}
$$

$$
\begin{aligned}
T_{a l} & =\frac{\left|\underline{V}_{r e f}\right| \sin \left(\frac{k \pi}{5}-\theta\right)}{\left|\underline{V}_{m}\right| \sin \left(\frac{\pi}{5}\right)}\left[\frac{\tau}{1+\tau^{2}}\right] T_{s} \\
T_{b m} & =\frac{\left|\underline{V}_{r e f}\right| \sin \left(\theta-\frac{(k-1) \pi}{5}\right)}{\left|\underline{V}_{m}\right| \sin \left(\frac{\pi}{5}\right)}\left[\frac{\tau}{1+\tau^{2}}\right] T_{s}
\end{aligned}
$$

The maximum value of the principal harmonic of the voltage that can be obtained using this SVPWM algorithm" is $85.41 \%$ of that obtained using only zero and large vectors. Therefore, it is about $0.5257 * \mathrm{~V}_{\mathrm{dc}}$ which is as shown in Eqs. (15).

$$
V_{\max }=0.8541 * \underline{V}_{l} * \cos (\pi / 10)=0.5257 * V_{d c}
$$

Regardless of the sector in which the reference vector is located, it is crucial to establish a correct switching sequence by determining the application order of the vectors. Switching sequence can be create in different ways for the best harmonic performance and low switching frequency for all inverters. When specifying the switching sequence, the transition from one switching state to the next should include only two switches on either leg of the inverter. At the same time, while one of the two switches on the same phase leg is on, the other must be off. In addition, to reduce the switching loss, the transition of the reference vector from one sector to the next should always be done with the least possible number of switching. Considering these situations, the switching sequence is very commonly used in which half of the switching period starts with one zero vector and ends with the other zero vector for a multi-phase inverter. This scheme is called Continuous SVPWM (CSVPWM). In Continuous SVPWM for a five-phase inverter, half of the switching period starts with zero vector $V_{0}$ and ends with other zero vector $V_{31}$.

In multi-phase inverters, switching losses are high at large powers due to the number of switches and therefore the number of switching. By connecting one or more inverter legs to the positive or negative DC-link in a switching period, the number of switching, i.e. switching losses, can be greatly reduced. This can be done by arranging the placement of the zero voltage vectors in a switching period. This kind of switching sequence is called discontinuous SVPWM (DSVPWM). There are six different discontinuous SVPWMs commonly used in the literature. The method where $T_{31}(11111)$ is kept zero for a switching period is called DPWMMIN, while the method where a $\mathrm{T}_{0}(00000)$ is kept zero is called DPWMMAX. In the DPWM0 scheme, $\mathrm{T}_{0}(00000)$ is kept zero in odd-numbered sectors and $\mathrm{T}_{31}(11111)$ in even-numbered sectors. DPWM1 is the opposite of DPWM0. In the DPWM2 scheme, each sector is divided into two $18^{\circ}$ segments. $\mathrm{T}_{0}(00000)$ in the first segment and $\mathrm{T}_{31}(11111)$ in the second segment are kept zero. DPWM3 is the opposite of DPWM2. Especially at low modulation indices, the DPWMAX scheme offers the lowest Total Harmonic Distortion (THD) and Weighted Total Harmonic Distortion (WTHD) values [13]. Therefore, in this article, the DPWMMAX scheme is chosen for the control of a five-phase inverter. In this study, the switching sequences created for Sector- 1 and Sector-2 using the DPWMMAX scheme are given in Figure 5. 


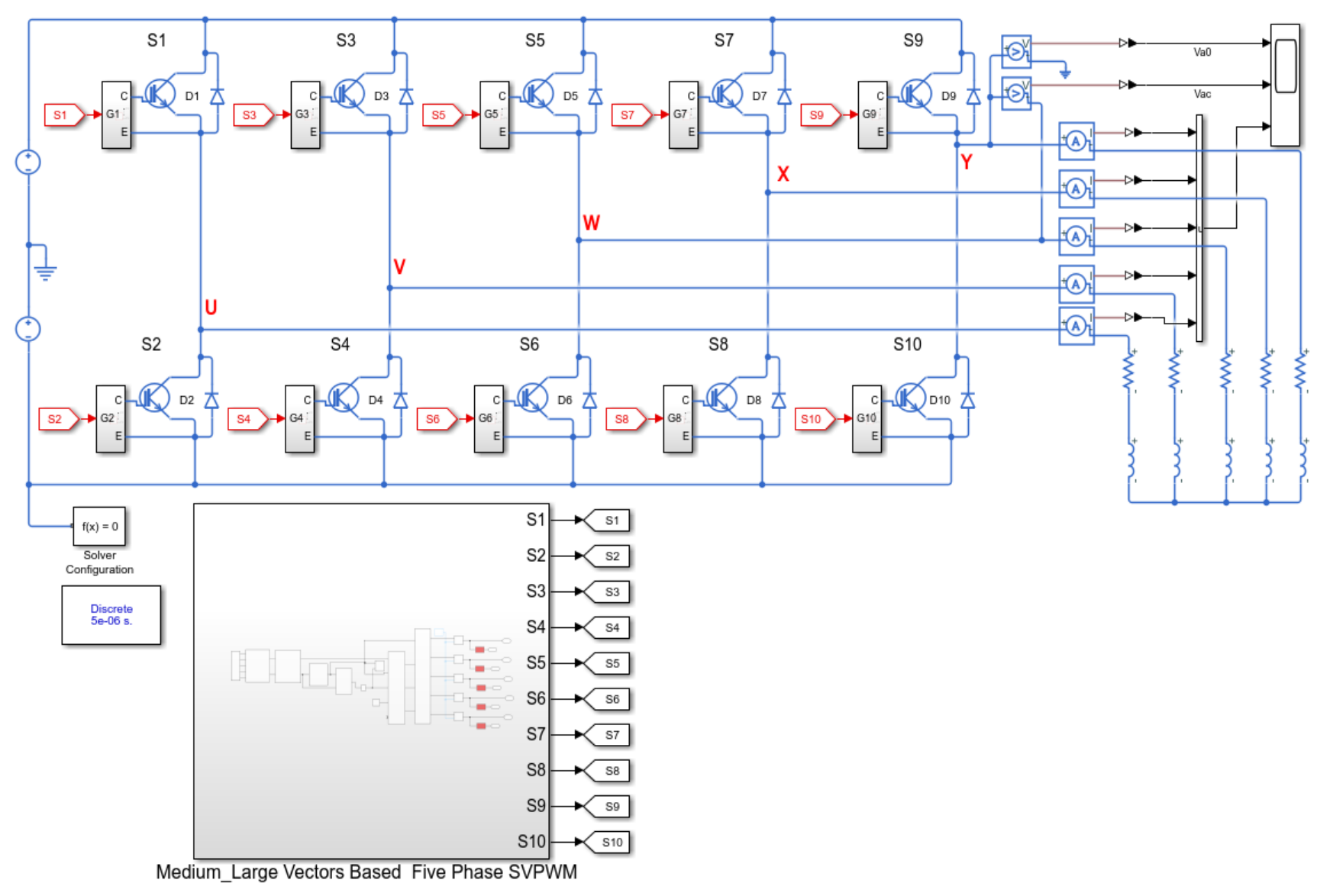

Figure 6. MATLAB simulation circuit of DSVPWM controlled five-phase inverter

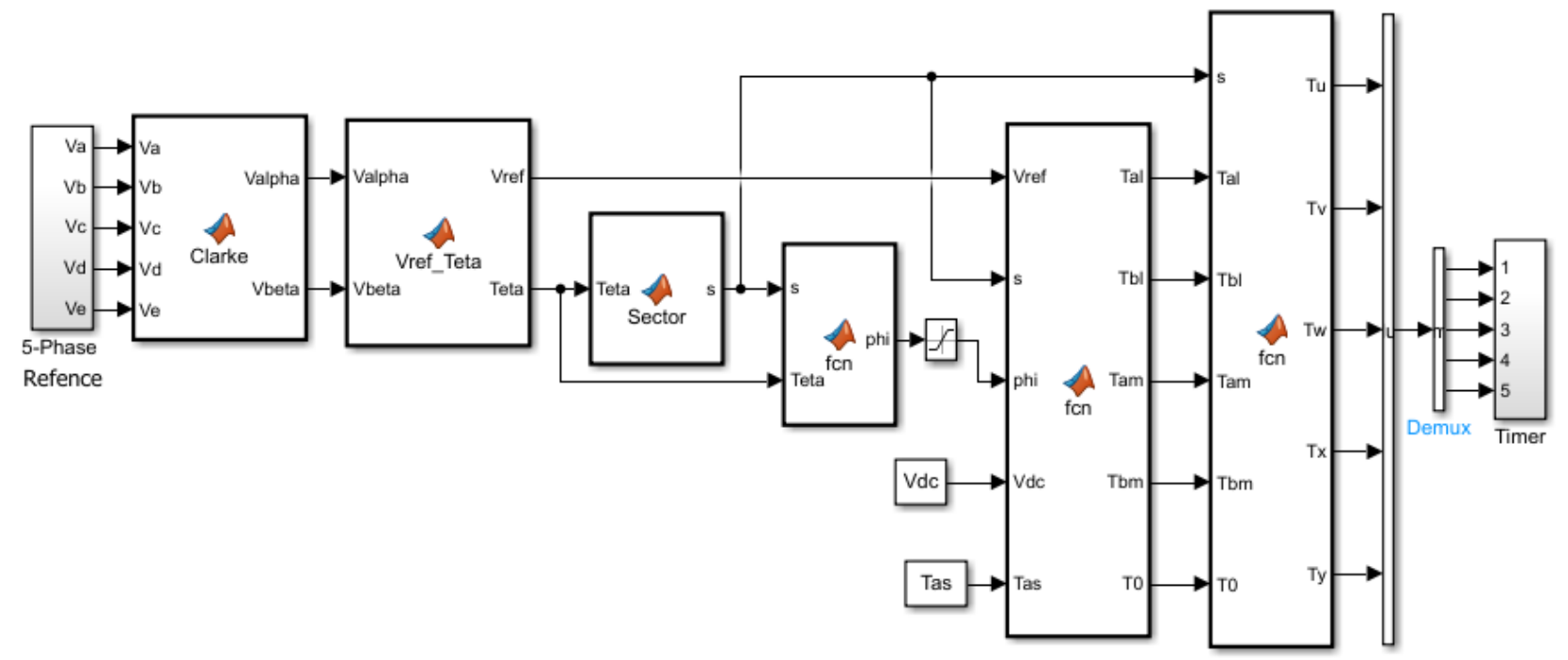

Figure 7. MATLAB algorithm of five-phase discontinuous SVPWM (DSVPWM) 


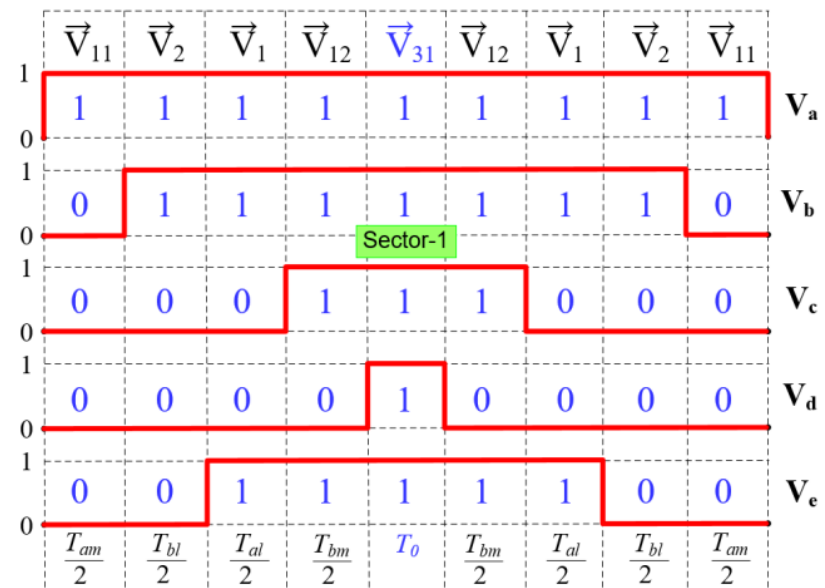

(a)

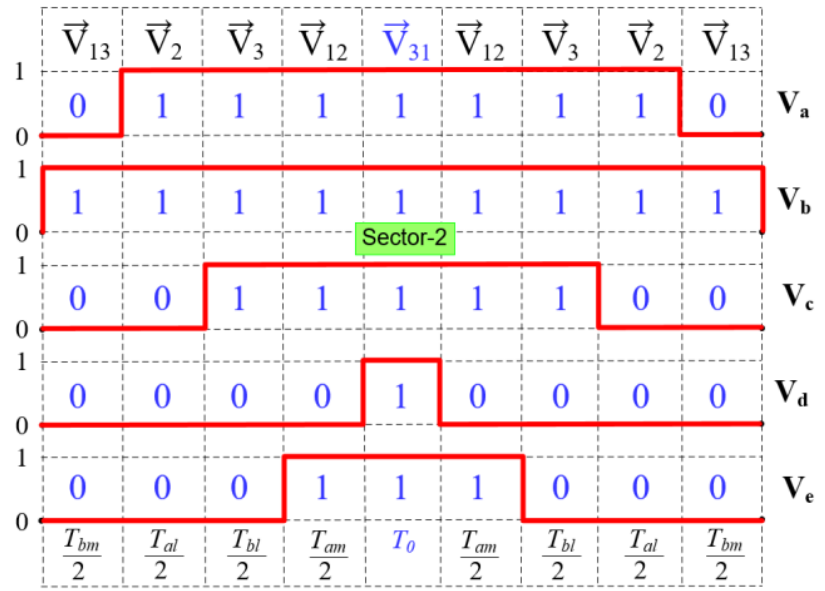

(b)

Figure 5. Switching sequence for (a) sector-1, (b) sector-2

\section{MATLAB SIMULATION OF FIVE PHASE INVERTER}

In this study, an SVPWM controlled five-phase two-level inverter feeding an RL load is simulated using MATLAB Simulink/Simscape blocks. The general view of the simulation circuit is given in Figure 6. The five-phase SVPWM algorithm developed for simulation is given in Figure 7. The SVPWM algorithm is created as software using MATLAB S-Function blocks, as seen in Figure 7. When calculating vectors dwell times, calculations are made by directing the $V_{\text {ref }}$ to Sector-1 regardless of which sector it falls into. Thus, complex calculations are reduced. The five-phase SVPWM algorithm using zero, medium and large vectors is run for 9-segment Discontinuous mode. The sampling time was taken as $\mathrm{T}_{\mathrm{s}}=5 \mu \mathrm{s}$. The dc-line voltage of the inverter is $V_{d c}=400 \mathrm{~V}$ and the output frequency is $\mathrm{f}=50 \mathrm{~Hz}$. The resistance and inductance value of the load is taken as $\mathrm{R}=20 \Omega, \mathrm{L}=40 \mathrm{mH}$. Simulation results are obtained for different values of modulation index and switching frequency. The waveforms are given below for only one case where the modulation index is $\mathrm{M}_{\mathrm{a}}=0.98$, the output frequency is $f=50 \mathrm{~Hz}$ and the switching frequency is $f_{s}=2250 \mathrm{~Hz}$. Other simulation results for different $\mathrm{M}_{\mathrm{a}}$ and $f_{s}$ are presented in Table 2.

For $\mathrm{M}_{\mathrm{a}}=0.98$, the five-phase modulation signals obtained from the five-phase DSVPWM algorithm are given in Figure 8 (a) and the turn-on time $\left(T_{u}\right)$ of the $S_{1}$ switch on the U-phase leg is given in Figure 8(b).

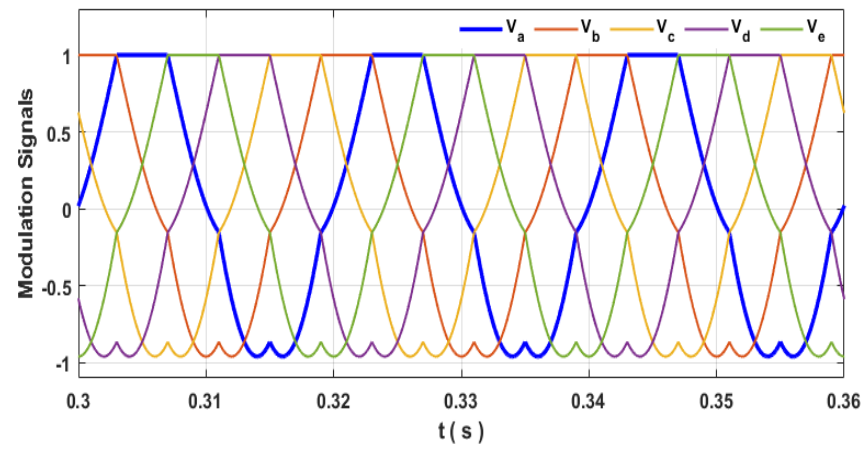

(a)

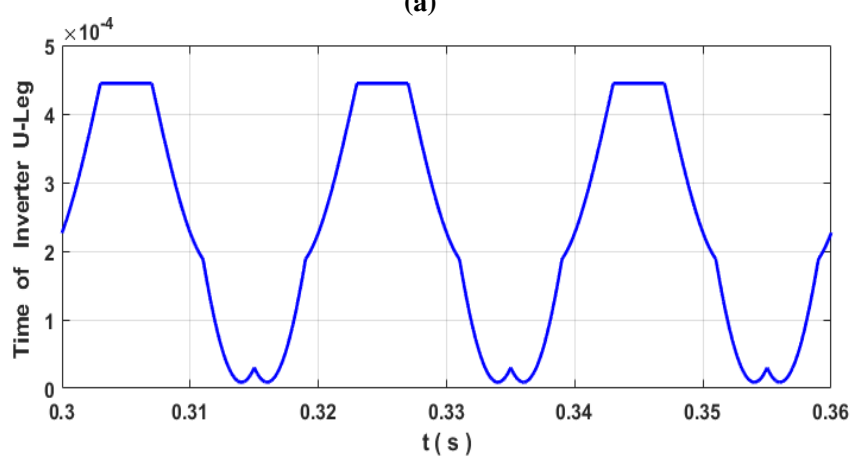

(b)

Figure 8. (a) 5-phase modulation signals, (b) the turn-on time (Tu) of the $\mathrm{S}_{1}$

The $\mathrm{V}_{\mathrm{a} 0}$ output voltage waveform of the U-phase leg of the five-phase inverter with respect to the midpoint of the DC-link and its harmonic analysis are given in Figure 9. As can be seen from Figure 9, the output phase voltage is a two-level voltage with $\pm \mathrm{V}_{\mathrm{dc}} / 2$ levels. For $\mathrm{M}_{\mathrm{a}}=0.98$, the amplitude of its fundamental harmonic is obtained as $206.10 \mathrm{~V}$. It is harmoniously with Equation 15. The \% THD value was calculated as $93.86 \%$.
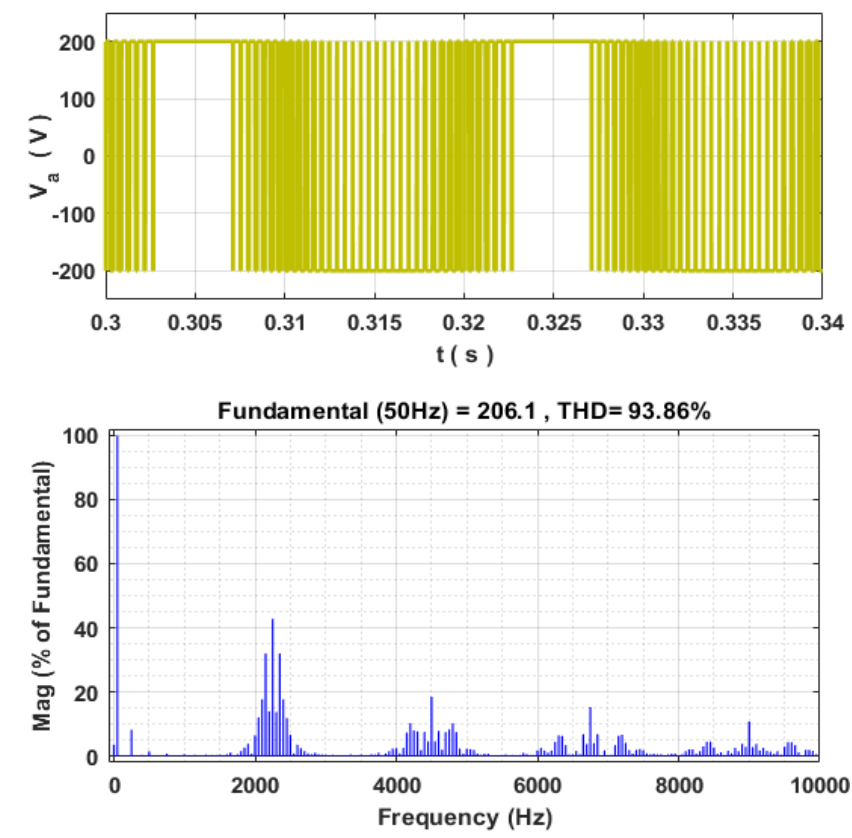

Figure 9. Output phase voltage and THD analysis of 5-phase inverter 

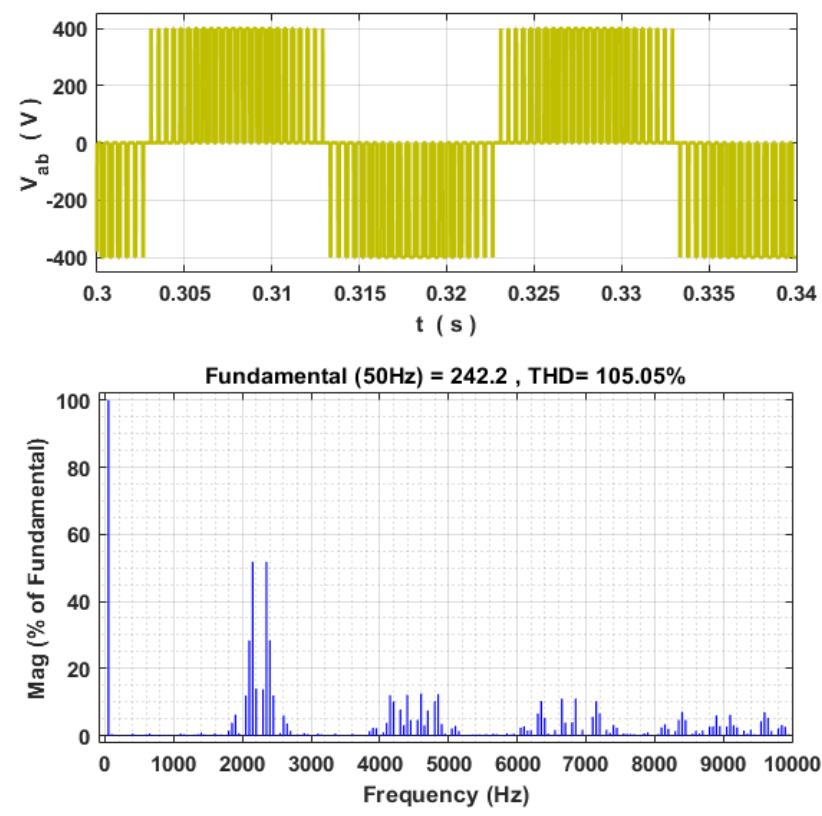

Figure 10. Output voltage and THD analysis between adjacent phase legs

The output voltage $V_{a b}$ between two adjacent phase legs of the SVPWM controlled five-phase inverter and its harmonic analysis are given in Figure 10. The output voltage $\mathrm{V}_{\mathrm{ac}}$ between two non-adjacent phase legs and its harmonic analysis are also given in Figure 11. As can be seen from Figure 10 and Figure 11 , all output phase-to-phase voltages are three-level voltage with $-\mathrm{V}_{\mathrm{dc}} / 2,0,+\mathrm{V}_{\mathrm{dc}} / 2$ levels. For $\mathrm{M}_{\mathrm{a}}=0.98$, the amplitudes of the fundamental component were obtained as $242.30 \mathrm{~V}$ $\left(\mathrm{V}_{\mathrm{a}} * 1.1756\right)$ for the $\mathrm{V}_{\mathrm{ab}}$ voltage and $392.10 \mathrm{~V}\left(\mathrm{~V}_{\mathrm{a}} * 1.9025\right)$ for the $\mathrm{V}_{\mathrm{ac}}$ voltage. These values are compatible with Equation 2 and Equation 3. It is clearly seen from the harmonic analysis that the line voltage $\mathrm{V}_{\mathrm{ab}}(105.02 \%)$ has a much higher harmonic content than the line voltage $\mathrm{V}_{\mathrm{ac}}(54.75 \%)$.
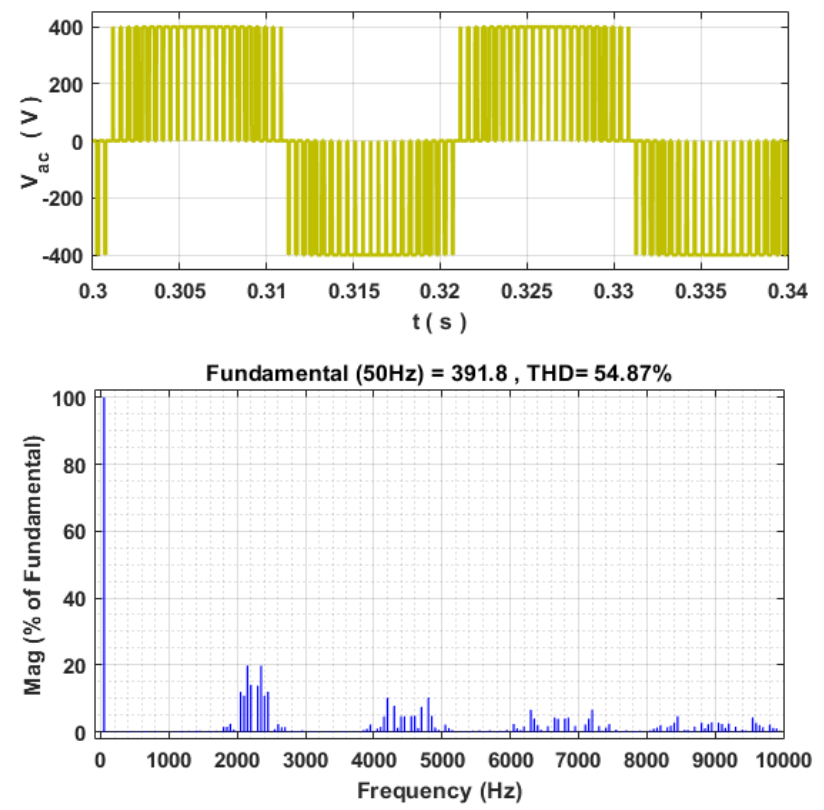

Figure 11. Output voltage and THD analysis between non-adjacent phase legs

The current waveform $I_{a}$ of the U-phase leg of the fivephase inverter and its harmonic analysis are given in Figure 12, and the five-phase current waveforms are also given in Figure 13. The phase current of the inverter, which feeds an RL load with $\mathrm{R}=20 \Omega, \mathrm{L}=40 \mathrm{mH}(\mathrm{Z}=23.62 \Omega)$ and whose phase voltage is $206.10 \mathrm{~V}$, is obtained as $8.728 \mathrm{~A}$. As can be seen from the harmonic analysis, it is an advantage that the phase current has a low THD rate of $2.53 \%$. This result is in accordance with the literature for the five-phase SVPWM technique using zero and four active vectors.
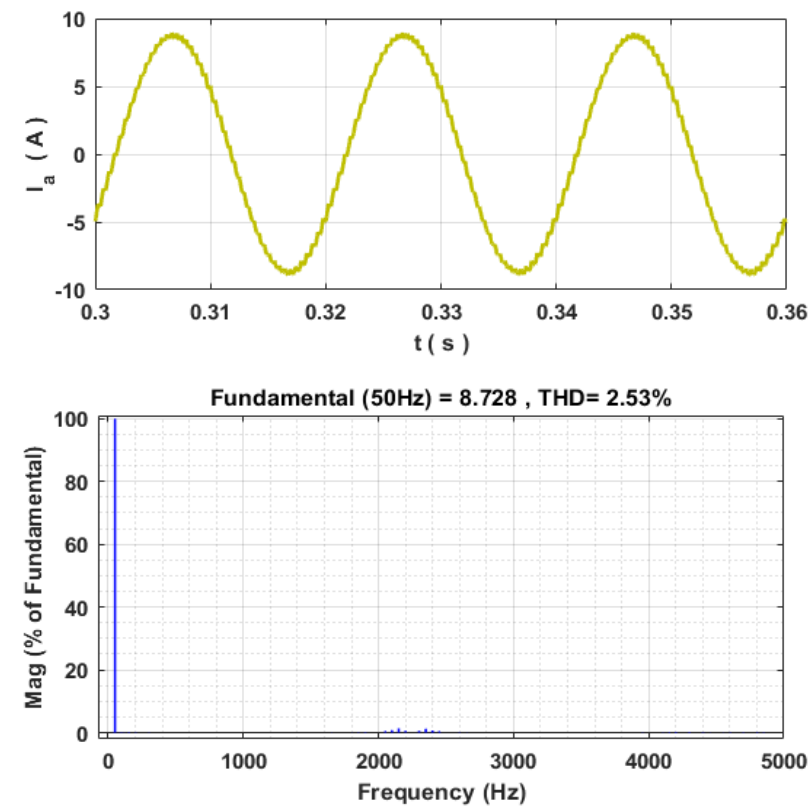

Figure 12. U-phase's current waveform and harmonic analysis

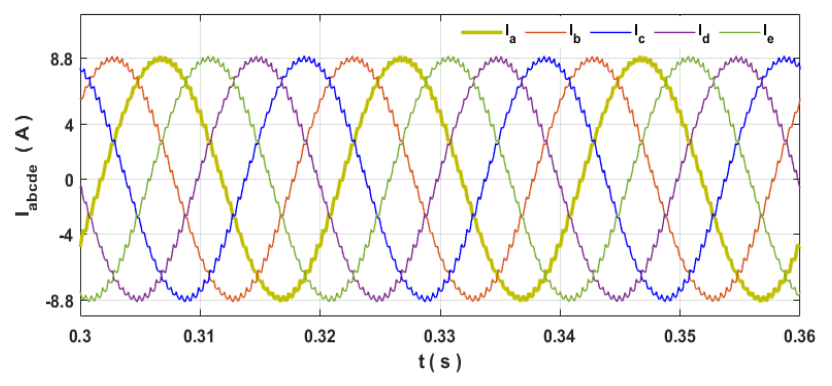

Figure 13. Current waveforms of the five-phase leg of the inverter

In addition to the results given above, the simulation is run for 0.7 and 0.4 values of the modulation index by keeping the switching frequency constant as $2250 \mathrm{~Hz}$. In addition, the simulation is run for the $5 \mathrm{kHz}$ and $10 \mathrm{kHz}$ values of the switching frequency by keeping the modulation index constant as 0.98 . All obtained voltage, current and their THD values are given in Table-2. The values in Table- 2 can be divided into two groups. The first is the case where $f_{s}$ is constant and $\mathrm{M}_{\mathrm{a}}$ is variable, and the second is the case where $\mathrm{M}_{\mathrm{a}}$ is constant and $f_{s}$ is variable. For constant $f_{s}$, as $\mathbf{M}_{\mathrm{a}}$ increases, the amplitudes of the fundamental components of all output voltages increase and the \% THD values decrease. In all cases, the amplitude of the voltage $\mathrm{V}_{\mathrm{ac}}$ is greater than $\mathrm{V}_{\mathrm{ab}}$ and the \% THD of $\mathrm{V}_{\mathrm{ac}}$ is smaller than $\mathrm{V}_{\mathrm{ab}}$. In general, the \% THD values of the voltages are very close to the 3-phase inverter. In addition, as the $\mathbf{M}_{\mathrm{a}}$ values increase, the \%THD values decrease. The amplitude value of the phase current fundamental component increases as $\mathrm{M}_{\mathrm{a}}$ increases. The \% THD value of the current is around 5\%, even at low $\mathbf{M}_{\mathrm{a}}$ values. In the second case, as $f_{s}$ increased for constant $\mathrm{M}_{\mathrm{a}}$, there is a slight decrease of $2-5 \mathrm{~V}$ in the amplitudes of the fundamental component of the output voltages. This is because the switching losses of IGBTs increase due to high $f_{s}$. While the 
TABLE II

SiMULATION RESULTS FOR DIFFERENT MODULATION INDEXES AND SWITCHING FREQUENCIES

\begin{tabular}{|c|c|c|c|c|c|c|c|c|c|c|}
\hline \multicolumn{11}{|c|}{ Medium and Large Vectors-9 Segment_DPWMMAX } \\
\hline No & $\mathbf{M}_{\mathbf{a}}$ & $f_{s}(\mathbf{H z})$ & $\mathbf{V}_{\mathrm{a} 0}(\mathbf{V})$ & $\mathbf{V}_{\mathbf{a}_{-} \text {THD }}$ & $\mathbf{V}_{\mathbf{a b}}(\mathbf{V})$ & $\mathbf{V}_{\text {ab_THD }}$ & $\mathbf{V}_{\text {ac }}(\mathbf{V})$ & $\mathbf{V}_{\text {ac_THD }}$ & $\mathbf{I}_{\mathbf{a}}(\mathbf{A})$ & $\mathbf{I}_{\mathbf{a} \_\mathbf{T H D}}$ \\
\hline 1 & 0.98 & 2250 & 206.10 & $\% 93.86$ & 242.30 & $\% 105.02$ & 392.1 & $\% 54.75$ & 8.728 & $\% 2.53$ \\
\hline 2 & 0.70 & 2250 & 147.40 & $\% 152.46$ & 173.50 & $\% 139.06$ & 280.60 & $\% 90.31$ & 6.246 & $\% 3.74$ \\
\hline 3 & 0.40 & 2250 & 84.29 & $\% 247.75$ & 99.28 & $\% 203.21$ & 160.60 & $\% 147.32$ & 3.574 & $\% 5.35$ \\
\hline 4 & 0.98 & $5 \mathrm{kHz}$ & 204.10 & $\% 95.65$ & 240.00 & $\% 105.44$ & 388.30 & $\% 56.03$ & 8.642 & $\% 1.27$ \\
\hline 5 & 0.98 & $10 \mathrm{kHz}$ & 202.10 & $\% 97.50$ & 237.60 & $\% 106.08$ & 384.50 & $\% 57.18$ & 8.558 & $\% 1.15$ \\
\hline
\end{tabular}

amplitude values of the phase current fundamental component are approximately constant, its \% THD values are much lower than $5 \%$. It is about $1.5-2.5 \%$, which is a good result.

\section{CONCLUSION}

There are two SVPWM methods for multi-phase VSI inverters. The first of these is the method in which only zero and large vectors are used. The second is the method in which zero, medium and large vectors are used. The method using zero and four active vectors provides output waveforms with lower THD from the inverter. Besides, there are two switching schemes in the SVPWM technique. These are called continuous and discontinuous SVPWM. The use of zero vectors for one switching period is reduced in the discontinuous SVPWM developed to reduce the switching losses in the inverter circuit. There are six different discontinuous SVPWM schemes in the literature. Of these, the DPWMMAX scheme provides lower THD even at low modulation indexes.

In this study, zero and four active vector-based SVPWM technique in discontinuous switching sequence (DPWMMAX) is proposed for a five-phase two-level VSI. The DSVPWM algorithm was created using MATLAB S-Function blocks. In addition, the complex calculations in the algorithm are reduced. To demonstrate the validity of the algorithm, a DSVPWMcontrolled five-phase inverter feeding an RL load is simulated in MATLAB/Simscape environment. The simulation results show the accuracy of the DSVPWM algorithm used.

\section{ACKNOWLEDGEMENT}

This work is supported by Firat University Scientific Research Projects (FUBAP) with TEKF.21.15 numbered project, titled "Development of Space Vector PWM (SVPWM) Switching Techniques Using Digital Signal Processor (DSP) for Different Inverter Types".

\section{REFERENCES}

[1] A. A. Al-Abduallah, et al., "Five-phase induction motor drive system with inverter output LC filter," presented at the $7^{\text {th }}$ IEEE GCC Conf. Exhibition, Doha, Qatar, Nov. 17-20, 2013.

[2] R. B. Shende, P. D. D. Dhawale, and P. K. B. Porate, "Modeling and simulation of five phase inverter fed im drive and three phase inverter fed im drive," International Journal of Engineering Research and Applications, vol. 1, no. 1, pp. 33-40, Jan. 2014.

[3] D. Raja and G. Ravi, "Design and implementation of five phase inverter with modified SVPWM switching technique for induction motor drive," presented at the Fifth Inter. Conf. on Science Technology Engineering and Mathematics, Chennai, India, Mar. 14-15, 2019.

[4] A. Pitrenas and A. Petrovas, "Six - phase VSI control using 8 - bit MCU," Balkan Journal of Electrical \& Computer Engineering, vol. 2, no. 3, pp. 104-107, Sept. 2014.
[5] A. Gundogdu and R. Celikel, "Performance analysis of open loop v/f control technique for six-phase induction motor fed by A multiphase inverter," Turkish J. Sci. Technol., vol. 15, no. 2, pp. 111-125, Sept. 2020.

[6] A. Lega et al., "General theory of space vector modulation for five-phase inverters," presented at the IEEE International Symposium on Industrial Electronics, Cambridge, UK, July 1-2, 2008.

[7] A. Bicak, "Analysis of direct torque control method of five-phase permanent magnet synchronous motor in electric vehicles," M.S. thesis, Dept. of Electrical and Electronics Engineering, Bursa Tech. Univ., Bursa, Turkey, 2018.

[8] S. Sadeghi et al., "Wide operational speed range of five-phase permanent magnet machines by using different stator winding configurations," IEEE Trans. Ind. Electron., vol. 59, no. 6, pp. 2621-2631, Apr. 2012.

[9] M. Tariq, "Five-phase induction motor drive system driven by five-phase packed u cell inverter: its modeling and performance evaluation," International Journal of Electrical and Computer Engineering, vol. 12, no. 9, pp. 664-669, May. 2018.

[10] O. Aydogmus, E. Deniz, and K. Kayisli, "PMSM drive fed by sliding mode controlled pfc boost converter," Arab. J. Sci. Eng., vol. 39, no. 6, pp. 4765-4773, June 2014.

[11] E. Deniz, "ANN-based MPPT algorithm for solar PMSM drive system fed by direct-connected PV array," Neural Comput \& Applic, vol. 28, pp. 3061-3072, Oct. 2017.

[12] M. A. Khan et al., "Analysis of discontinuous space vector PWM techniques for a seven-phase voltage source inverter," Int. J. Power Electronics Drive Syst., vol. 2, no. 2, pp. 203-218, June 2012.

[13] M. A. Khan, "Comprehensive analysis of discontinuous space vector pwm techniques for a five-phase voltage source inverter," Int. J. Power Electronics Drive Syst., vol. 1, no. 2, pp. 1-17, Apr. 2015.

[14] J. P. Corvalan, "Continuous and discontinuous modulation techniques for multiphase drives: analysis and contributions," Ph.D. thesis, Dept. Electronica Eng., Seville Univ., Seville, Spain, 2016.

[15] K. P. P. Rao, B. K. Veni, and D. Ravithej, "Five-leg inverter for fivephase supply," Int. J. Eng. Trends Technol, vol. 3, no. 2, pp. 144-152, Oct. 2012.

[16] M. T. Tessema, "V/f control design and simulation for five-phase induction motor," M.S. thesis, Dept. Electrical and Computer Eng., Addis Ababa Univ., Addis Ababa, Ethiopia, 2015.

[17] J. G. Haitham, Abu-Rub, and Atif Iqbal, High performance control of ac drives with matlab simulink. West Sussex, UK: Wiley, 2021.

[18] M. Narimani, and Bin Wu, High power converters and ac drivers. New Jersey, USA: Wiley, 2017.

[19] J. W. Kelly, E. G. Strangas, and J. M. Miller, "Multiphase space vector pulse width modulation," IEEE Trans. Energy Convers., vol. 18, no. 2, pp. 259-264, May 2013.

\section{BIOGRAPHIES}

Erkan Deniz was born in 1977. He received the B.S., M.S., and Ph.D. degrees from Firat University, Elazig, respectively in 2001, 2005, and 2011. From 2001 to 2012, he was a Research Assistant with the electrical teaching department. Since 2018, he has been an Associate Professor with the Electrical Electronics Engineering Department, Firat University. His research interests include power electronics, pulse width modulation techniques, power quality, STATCOM, energy storage systems, motor control. 